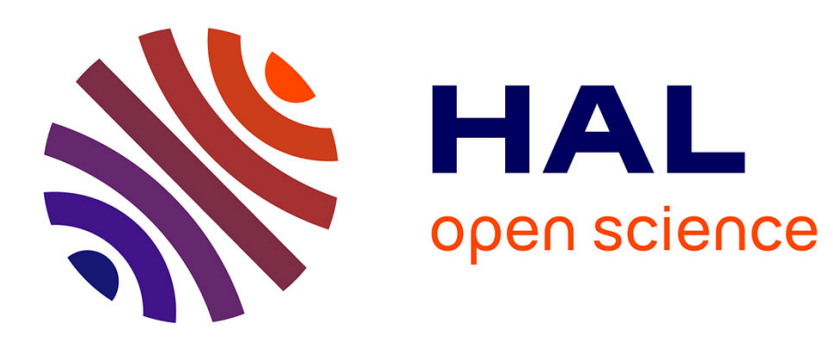

\title{
SVETLAN' or How to Classify Words Using Their Context
}

Gaël De Chalendar, Brigitte Grau

\section{To cite this version:}

Gaël De Chalendar, Brigitte Grau. SVETLAN' or How to Classify Words Using Their Context. Knowledge Acquisition, Modeling and Management, 12th International Conference, EKAW 2000, October 2-6, 2000, Proceedings, 2000, Juan-les-Pins, France. pp.203-216. hal-02458241

\section{HAL Id: hal-02458241 https://hal.science/hal-02458241}

Submitted on 28 Jan 2020

HAL is a multi-disciplinary open access archive for the deposit and dissemination of scientific research documents, whether they are published or not. The documents may come from teaching and research institutions in France or abroad, or from public or private research centers.
L'archive ouverte pluridisciplinaire HAL, est destinée au dépôt et à la diffusion de documents scientifiques de niveau recherche, publiés ou non, émanant des établissements d'enseignement et de recherche français ou étrangers, des laboratoires publics ou privés. 


\title{
SVETLAN' \\ or \\ How to Classify Words Using their Context
}

\author{
Gaël de Chalendar and Brigitte Grau \\ LIMSI/CNRS \\ BP 133, 91403 Orsay Cedex, France \\ \{gael, grau\}@limsi.fr
}

\begin{abstract}
Using semantic knowledge in NLP applications always improves their competence. Broad lexicons have been developed, but there are few resources which contain semantic information available for words and which are nondedicated to specialized domains. In order to build such a base, we designed a system, SVETLAN', able to learn categories of nouns from texts, whatever their domain. In order to avoid general classes mixing all the meanings of words, they are learned taking into account the contextual use of words.
\end{abstract}

\section{Introduction}

Improving the competence of NLP applications and Information Research systems requires to develop more and more in-depth analysis of documents in order to capture their meaning more precisely. In NLP, existing techniques correspond to two kinds of approaches, radically different. On one hand, surface analysis of texts is based on the distribution of words and their importance in a corpus. It exploits lexical knowledge on words and, possibly, general semantic information such as the semantic classes of a word and relations between words or concepts, as those found in WordNet [7] or in thesauri. This kind of approach can be applied to large text bases, whatever their subjects. In return, it does not allow these systems to develop a detailed and precise analysis, due to the lack of a precise and structured knowledge base. Even when using WordNet, this problem remains. Words in WordNet are related to a Synset when they are synonymous, however Synsets correspond to large categories, and there exists some shifts of meaning between two synonyms so that when two words belonging to a same Synset are considered within a specific context, they often no longer share a common meaning.

On the other hand, in-depth understanding systems require highly structured semantic knowledge as well as pragmatic knowledge about situations referred in texts (the events, their causal links and characters). These systems aim at developing semantic analysis of sentences and building a representation of the meaning of texts. Their limitation comes from these required resources that are impossible to build except in very limited and well-known domains. The problem we address in this paper is then to improve the first systems, in order to go towards the second ones, but without losing the large coverage of the first ones. This is done by automatically acquiring a 
more structured knowledge base than the bases presently used. Our goal is to extract knowledge from texts, considering that they contain many examples of word uses. We do not want to model a specialized domain, a priori chosen. On the contrary, we want to process "general" language, as opposed to a specialized language, but it is well known that there is no "general language corpus". Each corpus is a subpart of the language that has its own specificity. Thus we use a corpus made of newspaper articles (French Agence France Presse (AFP) newswires and "Le Monde" articles). This corpus covers a wide range of different subjects and so, is close to what would be a "general language corpus", apart from the quite fixed journalistic style.

Automatic processes that extract knowledge from texts belong to statistical or syntactical approaches with a lot of variations between these two extremes. Purely statistical ones, such as [15] often obtain groups of words that characterize a sense of a target word. Other approaches ([1], [6], [10], [11], [14]) are more syntactical and obtain classes of words with similar meanings ${ }^{1}$ : ARIOSTO [1] and STARTEX [14] use manual semantic tagging and next classification, but while STARTEX uses not tagged repeated text segments, ARIOSTO uses syntactically parsed text. On the contrary ASIUM [6] applies a cooperative clustering algorithm on classes obtained from syntactically parsed texts. ZELLIG [11] constructs a graph of words linked by their common contexts in elementary noun phrases. By the study of the characteristics of this graph, it builds semantic categories. These three systems are designed to work on specialized languages belonging to a specific domain, whose vocabulary is well defined and generally not very ambiguous. When applied to non-specialized texts, they lead to build large classes, mixing all the meanings of words ${ }^{2}$, as shown by Fabre et al. [5] for ZELLIG.

As newspapers articles contain a lot of polysemous words, these approaches cannot be applied directly, because they are more or less based on the assumption that their target corpus is sufficiently specialized to be able to ignore the problem of polysemy. However, when considering these polysemous words in their contextual use, i.e. a segment of text about only one topic, their meaning is no more ambiguous. Thus, our system, SVETLAN', takes advantage of a precise context recognition ability in order to build homogeneous classes of words despite the generality of the corpus. It is based on a distributional approach: nouns playing the same syntactic role with a verb in sentences related to the same topic, i.e. the same domain, are aggregated in the same class. The contextual approach of SVETLAN' relies on knowledge about semantic domains of texts, automatically learned by SEGAPSITH [9]. This is way of focusing the construction of the classes by reference to controlled topics. It allows our system to deal naturally with more general texts while giving classes of words with similar meanings, in comparison with the other approaches which have to process specialized texts or that give more disjoined classes.

${ }^{1}$ In the rest of this paper, we use the term 'words with similar meanings' to identify words that refer to concepts with an immediate, or quasi-immediate, ancestor in a conceptual ontology.

${ }^{2}$ Pereira et al. [13] use also syntactically parsed texts and a statistical clustering algorithm but applied to more general texts: Associated Press newswires. Classes obtained do not seem to constitute synonym sets as those of the preceding systems, but they have been used successively on a disambiguation task. 


\section{Overview of the System}

Input data of SVETLAN' (see Fig. 1) are semantic domains with the Thematic Units (TUs) that have given birth to them. TUs are the result of a topic segmentation process relying on lexical cohesion that processes texts such as newspaper articles. Each TU contains the lemmatized content words of a text segment. The domains are automatically learned by aggregating similar thematic units. So, domains are sets of weighted words, relevant to represent a specific topic.

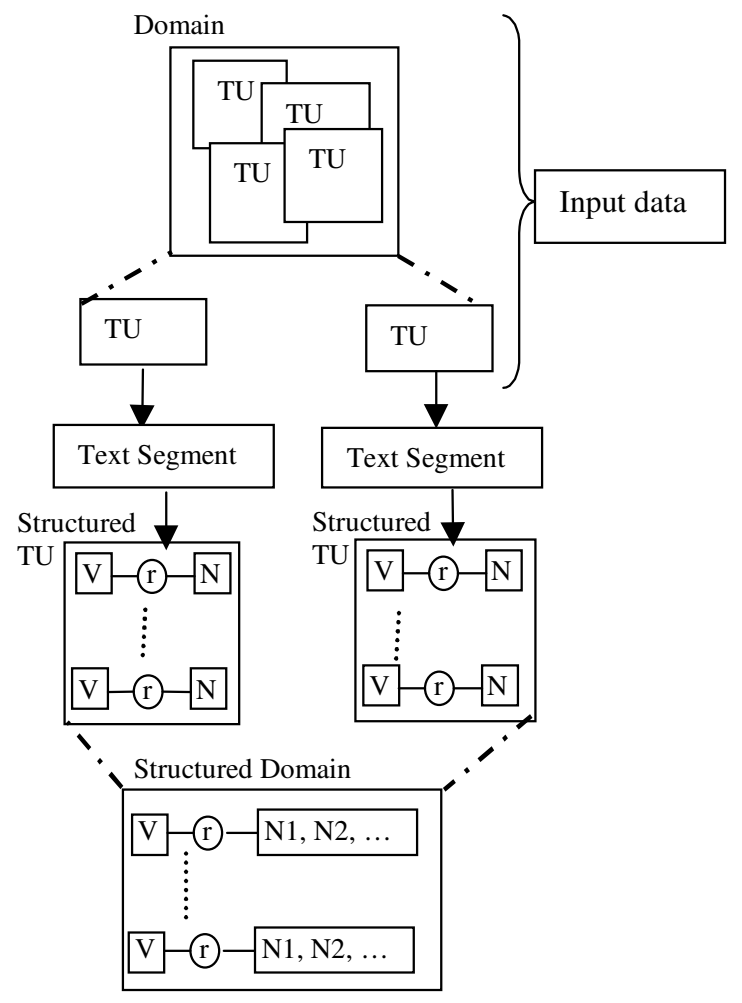

Fig. 1. Schema of structured domain learning

The first step of SVETLAN' consists of retrieving text segments of the original texts associated to the different TUs in order to parse their sentences. SVETLAN' extracts triplets from the parser results, that are constituted by a verb, the head noun of a phrase and its syntactic role. Thus, to each TU corresponds a text segment and a set of triplets we call a structured thematic unit. In order to build classes of nouns according to their contextual use, only the structured thematic units associated to a same semantic domain are aggregated altogether. Aggregation consists of grouping nouns playing the same syntactic role with a same verb. In such an approach, a verb associated to a type of phrase (subject, direct object, ...) constitutes a criterion for 
building categories. However, one can find words belonging to different semantic categories associated to a same verb and a same type of phrase when the verb is polysemous, as for example to drive a car and to drive a railway; the same problem occurs with very general verbs, as to search for example, that do not entail alone a discrimination among their direct objects. Thus the application of a distributional method without any control coming from the context would lead the system to build heterogeneous categories. SVETLAN' solves this problem by aggregating verb complements within TUs belonging to a same domain. That ensures a better homogeneity of the data and leads SVETLAN' to form context sensitive classes. A last filtering step, based on the weights of the words in their domain, allows the system to eliminate nouns from classes when they are not very relevant in this context. This whole process leads to the formation of structured domains, i.e. semantic domains where each verb is related to categories of nouns that are semantically homogenous.

\section{Semantic Domain Learning}

We only give here a brief overview of the semantic domain learning module. This one is described more precisely in [9]. This module incrementally builds topic representations, made of weighted words, from discourse segments delimited by SEGCOHLEX [8]. It works without any a priori classification or hand-coded pieces of knowledge. Processed texts are typically French newspaper articles coming from $L e$ Monde or AFP. They are pre-processed to only keep their lemmatized content words (adjectives, single or compound nouns and verbs).

The topic segmentation implemented by SEGCOHLEX is based on a large collocation network, built from 24 months of Le Monde newspaper, where a link between two words aims at capturing semantic and pragmatic relations between them. The strength of such a link is evaluated by the mutual information between its two words. The segmentation process relies on these links for computing a cohesion value for each position of a text. It assumes that a discourse segment is a part of text whose words refer to the same topic, that is, words are strongly linked to each other in the collocation network and yield a high cohesion value. On the contrary, low cohesion values indicate topic shifts. After delimiting segments by an automatic analysis of the cohesion graph, only highly cohesive segments, named Thematic Units (TUs), are kept to learn topic representations. This segmentation method entails a text to be decomposed in small thematic units, whose size is equivalent to a paragraph. Discourse segments, even related to the same topic, often develop different points of view. To enrich the particular description given by a text, we add to TUs those words of the collocation network that are particularly linked to the words found in the corresponding segment.

Learning a complete description of a topic consists of merging all successive points of view, i.e. similar TUs, into a single memorized thematic unit, called a semantic domain, or simply a domain. Each aggregation of a new TU increases the system's knowledge about one topic by reinforcing recurrent words and adding new ones. Weights on words represent the importance of each word relative to the topic and are computed from the number of occurrences of these words in the TUs. This method leads SEGAPSITH to learn specific topic representations as opposed to [12] for 
example whose method builds general topic descriptions as for economy, sport, etc.

\begin{tabular}{|l|l|c|c|}
\hline \multicolumn{1}{|c|}{ mots } & \multicolumn{1}{|c|}{ words } & occ. & weight \\
\hline juge d'instruction & examining judge & 58 & 0.501 \\
détention & police custody & 50 & 0.442 \\
bien public & public property & 46 & 0.428 \\
charge & charging & 49 & 0.421 \\
emprisonner & to imprison & 45 & 0.417 \\
cour d'appel & court of criminal appeal & 47 & 0.412 \\
recel & receiving stolen goods & 42 & 0.397 \\
supposer & to presume & 45 & 0.382 \\
police judiciaire & criminal investigation department & 42 & 0.381 \\
fraude & fraud & 42 & 0.381 \\
\hline
\end{tabular}

Fig. 2. The most representative words of a domain about justice

We have applied the learning module of SEGAPSITH on one month (May 1994) of AFP newswires. Fig. 2 shows an example of a domain about justice that gathers 69 TUs. In all figures displaying examples extracted from our results obtained on French texts, we will put the original data plus their literal English translation.

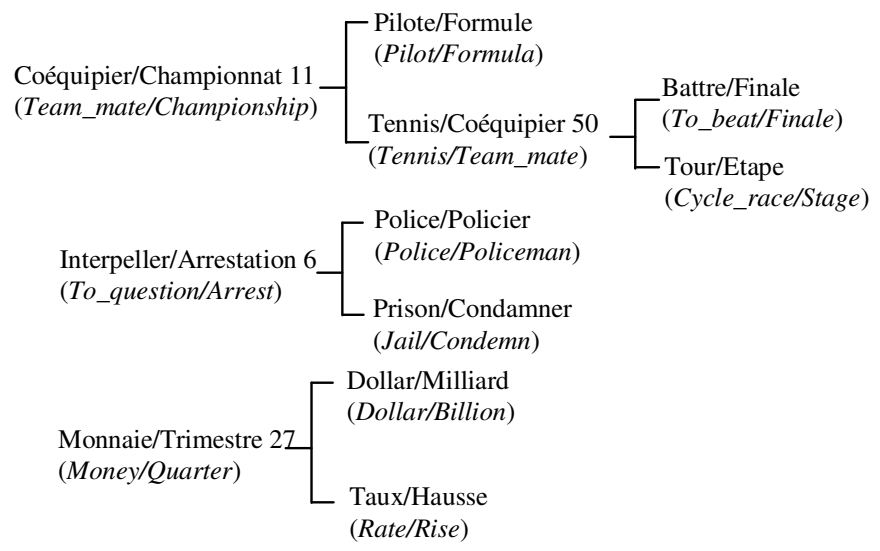

Fig. 3. Three hierarchies of semantic domains

As some of these domains are close and refer to the same general topic, we have applied a hierarchical classification method based on their common words to organize them in separate general topics and to structure them. Fig. 3 shows the hierarchies built about sport, police and stock exchange. Each leaf is a domain, named by its two more weighted words, while their name and their size, i.e. the number of common words found in their children, describe internal nodes.

\section{Structured Domains Learning}

As in [6], verbs allow us to categorize nouns. Those nouns that play a same role 
relative to a same verb define a class. In order to learn very homogeneous ${ }^{3}$ classes, we only apply this principle on words belonging to a same context, i.e. a domain.

\subsection{Syntactic Analysis}

In order to find the verbs and their arguments in the texts, we use the syntactic analyzer Sylex $[3,4]$.

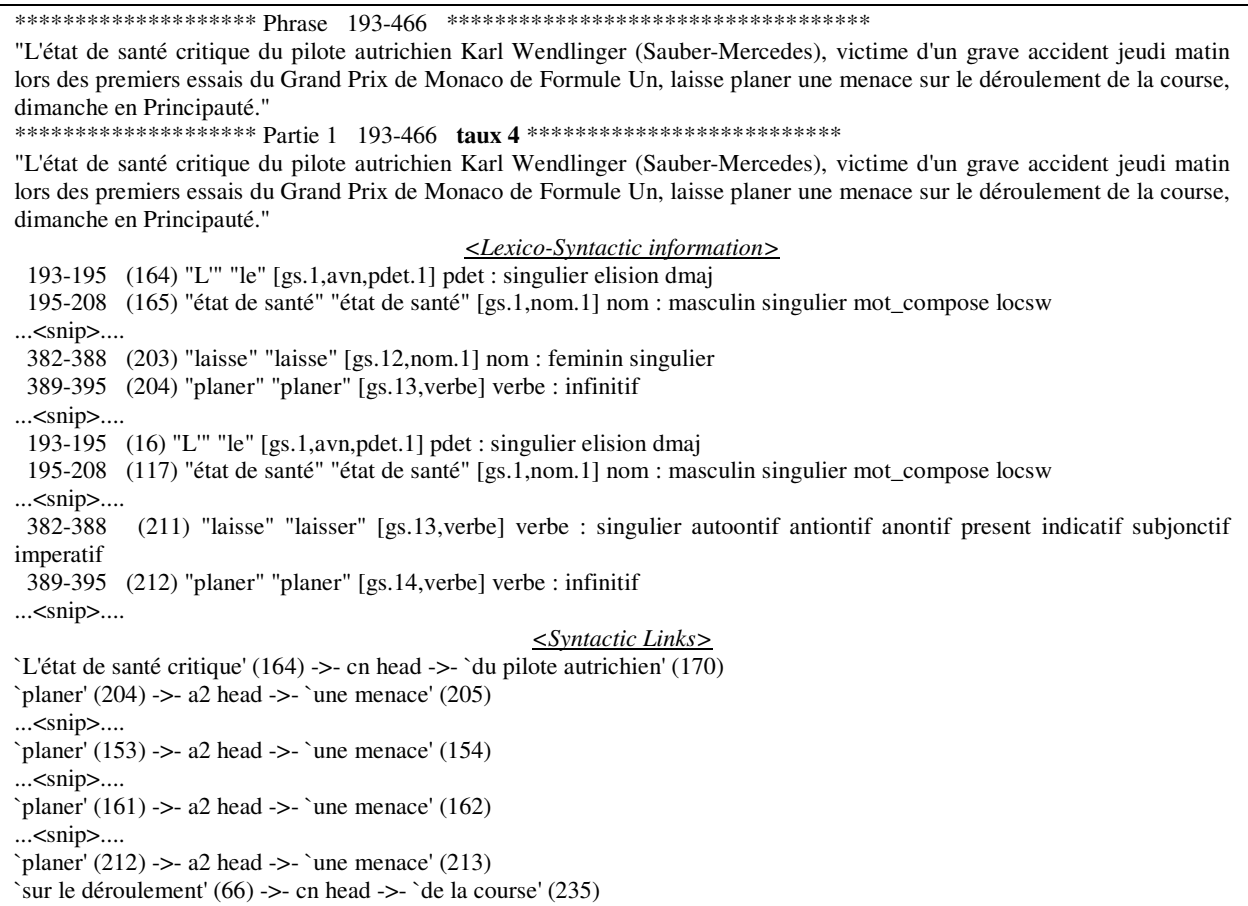

Fig. 4. An extract of an analysis of a sentence by Sylex (in French only).

Fig. 4 shows a little part of the results of Sylex for a sentence. The first part exhibits lexico-syntactic information for the words and this for four different interpretations (indicated by the code "taux 4" = rate 4) due to the fact that Sylex cannot chose between two interpretations for two words: "laisse" between the verb "laisser" (to let) and the noun "laisse" (leash) and "critique" between the verb "critiquer" (to criticize) and the adjective "critique" (critical). The second part shows the syntactic links found by Sylex. Between parenthesis are references to the words in the preceding analysis. Here Sylex has found four times the same interpretation. In this case, we count one occurrence of the link. But if it finds several times the same relation between a verb and different words, for example several possible subjects, then we keep all the different interpretations. That is because we have no way to choose between them. We

${ }^{3}$ We call homogeneous a class that contains words that have similar meanings, as defined above, in the corresponding domain. 
make the reasonable expectation that the false interpretations will have much less occurrences in the corpus and so, will be filtered out during the rest of the processing.

The results of Sylex are very detailed and not easy to parse directly with, say, Perl. Furthermore, we do not need all the information it extracts. In fact, we only need to find the verb with its links and the head nouns arguments of these links. So, we have developed a formal grammar that extracts from these raw analyzes the associations between a verb and its arguments. This grammar extracts links from the results of Sylex in the following format:

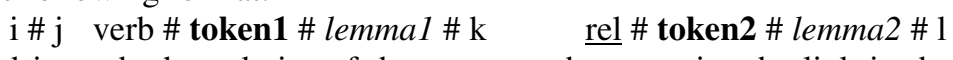

where $\mathrm{i}$ and $\mathrm{j}$ are the boundaries of the sentence that contains the link in the corpus; token 1 and lemmal are the token and the lemma of the verb respectively; rel is the syntactic relation which can be "subject", "direct object" or a preposition ("to", "from", etc.) ; token 2 and lemma 2 are the token and the lemma of the head noun of the noun phrase pointed by the relation; lastly, $k$ and $l$ are the indexes in the corpus of token 1 and token 2 respectively. Fig. 5 shows some links that we have extracted.

\begin{tabular}{|c|c|c|c|c|}
\hline token 1 & lemme 1 & relation & token 2 & lemme 2 \\
\hline plane & planer & sujet & menace & menace \\
\hline joue & jouer & COD & coupe & coupe \\
\hline apprenons & apprendre & de & sources & source \\
\hline token1 & lemmal & rel & token2 & lemma2 \\
\hline hang over & hang over & subject & threat & threat \\
\hline play & play & object & cup & cup \\
\hline hear & hear & of & sources & source \\
\hline
\end{tabular}

Fig. 5. Examples of extracted links

Sylex, as other syntactic analyzers, has problems with some constructions and consequently introduces errors that can cause problems to the remaining of the system. Some common errors are the bad interpretation of the passive form that causes a subject to be analyzed as a direct object and conversely, a direct object to be viewed as a subject. Another common error is that it often happens that Sylex does not find any link in a phrase. That is what we call silence. We will see in Sect. 5 that we can obtain good results despite these problems thanks to the redundancy needed to validate the links in the next steps of processing. But a consequence of this redundancy need is that the system must use great quantities of texts in order to create classes with a satisfactory size.

Having obtained the syntactic links that were in the texts, we want to group them relatively to the belonging of their text segment to a Thematic Unit. So, we define a structured thematic unit as a set of $<$ Verb $\rightarrow$ syntactic relation $\rightarrow$ Noun $>$ structures, syntactic relations instantiated with a verb and a noun. We will refer to these structures as instantiated syntactic relations. We are able to put in relation the links extracted from the results of Sylex and the words contained in the domains because each domain in the thematic memory remembers which thematic units have been used to create it. In the same way, each thematic unit remembers the part of text it comes from.

\subsection{Aggregation}

In order to construct groups of words with very similar meanings, we want to group the 
nouns appearing with the same syntactic role in relation to a verb inside a domain. Then, a structured domain is a set of $\left\langle\right.$ Verb $\rightarrow$ syntactic relation $\rightarrow$ Noun $_{1}, \ldots$, Noun $\left._{\mathrm{n}}\right\rangle$ structures, i.e. a set of aggregated instantiated syntactic relation.

Structured thematic units related to a same domain are aggregated altogether to form the structured domains. Aggregating a structured thematic unit within a structured domain consists of:

- aggregating their instantiated syntactic relations that contain a same verb ;

- adding new instantiated syntactic relations, i.e. adding new verbs with their arguments made of a syntactic relation and the lemmatized form of a noun.

\begin{tabular}{|c|c|c|}
\hline \multicolumn{3}{|c|}{ Structured Domain source } \\
\hline \multirow[t]{2}{*}{ to play [4] } & object & cup [3], match [1] \\
\hline & with & ball [1] \\
\hline \multirow[t]{2}{*}{ to win [2] } & subject & player [1] \\
\hline & object & match [1] \\
\hline \multicolumn{3}{|c|}{2 Instantiated Syntactic Relations sources } \\
\hline to play & \begin{tabular}{|l|} 
subject \\
object
\end{tabular} & $\begin{array}{l}\text { champion } \\
\text { match }\end{array}$ \\
\hline to lose & object & championship \\
\hline
\end{tabular}

\begin{tabular}{||l|l|l||}
\hline \hline Structured Domain result & \\
\hline \multirow{3}{*}{ to play [5] } & object & cup [3], match [2] \\
\cline { 2 - 3 } & with & ball [1] \\
\cline { 2 - 3 } & subject & champion [1] \\
\hline \multirow{2}{*}{ to win [2] } & subject & player [1] \\
\cline { 2 - 3 } & object & match [1] \\
\hline \hline & object & championship [1] \\
\hline
\end{tabular}

Fig. 6. An example of the aggregation of an instantiated syntactic relation in a structured domain

Fig. 6 shows the aggregation of a structured domain and two instantiated syntactic relations. This example shows all the possible effects of the aggregation. In the figure, bold elements represent new or updated data. Aggregating an instantiated syntactic relation in a structured domain that already contains the verb of the instantiated syntactic relation leads to increment the occurrence number of the verb, as for play in the example which occurred 4 times before the aggregation. Similarly, the occurrence number of common nouns related to the verb by the same relation are updated (match goes from 1 to 2), and new relations with their associated nouns are added to the verb. In the example, the subject champion with an occurrence of 1 is added. An instantiated syntactic relation with a new verb is simply added with an occurrence of 1 , as for $<$ to lose $\rightarrow$ object $\rightarrow$ championship $>$.

Classes of nouns in the produced structured domains contain a lot of words that disturb their homogeneity. These words often belong to parts of the various TUs at the origin of the structured domain that are not very related to the described topic. They correspond to a meaning of a verb scarcely used in the context defined by the domain. Another possibility is that the instantiated syntactic relation results from an error of Sylex. As these words are weekly weighted in the corresponding domains, the data can be filtered: each noun that possesses a weight lower than a threshold is removed from 
the class. By this selection, we reinforce learning classes of words according to their contextual use. At this time the threshold is empirically chosen. In the future, we plan to automatically find the threshold. It would be fixed in order to maximize the score of a validation task we outline in Sect. 7 . Fig. 7 shows two aggregated links obtained without filtering in its upper part and the filtered counterparts in its lower part. The link for the verb 'to establish' has been completely removed while the link of the verb 'to answer' with the preposition 'to' has been reduced by the removing of 'list'. We can see on this example that this filtering is efficient: the verb 'to establish' is not very related to the domain of 'nuclear weapons' from which this example is taken and the usage of 'to answer to a list' has a very low probability. More details on the effects of the filtering will be given in the Results section.

\begin{tabular}{|l}
\begin{tabular}{|lll|}
\hline $\begin{array}{l}\text { établir } \\
\text { répondre }\end{array}$ & $\begin{array}{l}C O D \\
a\end{array}$ & $\begin{array}{l}\text { base, zone } \\
\text { document, question, liste }\end{array}$ \\
\hline $\begin{array}{l}\text { établir } \\
\text { répondre }\end{array}$ & à & $\begin{array}{l}\text { base, zone } \\
\text { document, question, liste }\end{array}$ \\
\hline \begin{tabular}{lll}
\hline to establish \\
to answer
\end{tabular} & $\begin{array}{l}\text { object } \\
\text { to }\end{array}$ & $\begin{array}{l}\text { base, zone } \\
\text { document, question, list }\end{array}$ \\
\hline $\begin{array}{l}\text { to establish } \\
\text { to answer }\end{array}$ & $\begin{array}{l}\text { object } \\
\text { to }\end{array}$ & base, zone \\
document, question, tist
\end{tabular} \\
\hline
\end{tabular}

Fig. 7. Two filtered aggregated links in a domain about nuclear weapons

In the principle, the described operations are not very complicated. The difficulties come from the necessity to work with data coming from various tools. Furthermore, for performance and practical reasons, we do not apply the chain of tools text by text. The natural way to see the process would be to:

- read a text,

- extract the TUs from it,

- extract the corresponding structured thematic units,

- add each TU to its domain,

- add each structured thematic unit to its corresponding structured domain.

In fact, each computing step is done on the entire corpus and the results are next aligned. This allows us to save computation time because we do not have to run each one multiple times. But in return we have to deal with dictionaries and indexes for various files and tools.

\section{$5 \quad$ Results}

The experiments we realized aim at showing that SVETLAN' lead to learn classes of words which have similar meanings in the domain. To obtain such results we have chosen to run our system on one month of the French version of AFP wires, that forms a corpus stylistically coherent but that covers varied subjects with very polysemous and non specific verbs.

The judgements about the quality of the classes obtained are now manual and made 
by ourselves. We will obviously need a better and more systematic validation approach in the future. We will sketch in Sect. 7 some possibilities we consider.

The one month of $A F P$ wires is made of 4,500,000 words and 48,000 sentences in 6,000 texts. The thematic analysis gives 8,000 TUs aggregated in 2,000 domains. More details on these domains can be found in [9]. From these 48,000 sentences, Sylex extracts 117,000 different instantiated syntactic relations. 24,000 of these links concern subject, direct object, or circumstantial complements introduced by a preposition and are integrated in 1,531 structured domains.

After aggregating, but before filtering, the system obtains 431 aggregated links with two or more arguments, equivalent to 431 word classes. Some of them, such as <to manufacture $\rightarrow$ direct object $\rightarrow$ bomb, weapon $>$ are good. Nevertheless other classes are heterogeneous as <to return $\rightarrow$ direct object $\rightarrow$ territory, strip, context, synagogue $>$ (here strip comes from the Gaza Strip), or clearly mix different meanings of a verb, like <to quit $\rightarrow$ direct object $\rightarrow$ base, government $>$ which mixes together the meanings "to leave a place" and "to retire from an institution". For the two latter cases, one can see the interest to take into account the fact that the domains contain words with different weights representing their relevance to this domain. The higher the weight, the higher the relevance of this word in this domain. So we apply the aforesaid filter to our classes and retain only those with weights higher than a threshold. The class <territory, strip, context, synagogue $>$ is corrected to <territory, strip $>$ and $<$ base, government $>$ is removed.

Among the wrong classes, some are due to errors of Sylex, as $<$ to confer $\rightarrow$ direct object $\rightarrow$ price, actor $>$ where actor should be linked to to confer by the preposition to. The remaining errors are due to the extensive use of two different meanings of the verb in the same domain, as for: <to conduct/to manage $\rightarrow$ direct object $\rightarrow$ delegation, negotiation > (in French: "conduire une négociation/une délégation"). This kind of error is inherent to the method we use and should be removed by other means. Note again that the correctness of the links have been manually judged by ourselves.

We have tried two thresholds: 0.05 and 0.1. Fig. 8 details the results for both.

\begin{tabular}{|l|r|c|c|cc|}
\hline Threshold & Total & Good & Sylex errors & \multicolumn{2}{|c|}{ Remaining errors } \\
\hline $\mathbf{0 . 0 5}$ & 73 & 46 & 13 & \multicolumn{2}{|c|}{14} \\
& & $63 \%$ & $18 \%$ & \multicolumn{2}{c|}{$19 \%$} \\
\hline $\mathbf{0 . 1}$ & 38 & 27 & 7 & 4 & $11 \%$ \\
\hline
\end{tabular}

Fig. 8. Results of the filtering for two thresholds

After filtering, a lot of classes are removed but the remaining classes are well founded in most cases. An example of a retained class for both thresholds is: $<$ to injure $\rightarrow$ subject $\rightarrow$ colonist, soldier $>$

With a threshold set to 0.1 rather than to 0.05 , we retain only 38 links, but we gain $8 \%$ in precision. If we ignore the errors due to Sylex, the real precision of SVETLAN' is in the first case $78 \%$ and in the second case $87 \%$. It shows the interest there is to choose a good threshold.

Our experiments lead to homogeneous classes containing words having similar meanings, though these classes contain few words. In order to show the efficiency of building classes of words inside a domain, it is interesting to see what kind of classes would be obtained by the merging of all domains, that is to say: creating context-free 
classes. So, we have applied the same aggregation principle to the same corpus but without taking into account the domains. Just below, we show two classes for the verb "to replace". The top one is made context-free and the bottom one is made inside a domain. This verb is very general, virtually everything can be replaced.

\begin{tabular}{|l|l|l|}
\hline remplacer & $C O D$ & $\begin{array}{l}\text { texte, constitution, pantalon, combustible, loi, dinar, barre, } \\
\text { film, circulation, juge, saison, appareil, parlement, bataillon, } \\
\text { police, président, traité }\end{array}$ \\
\hline remplacer & COD & combustible, barre \\
\hline to replace & object & $\begin{array}{l}\text { text, constitution, trousers, combustible, law, dinar, bar, film, } \\
\text { circulation, judge, season, device, parliament, battalion, } \\
\text { police, president, treaty }\end{array}$ \\
\hline to replace & object & combustible, bar \\
\hline
\end{tabular}

The first group of words merges very different senses while the second class, much more little, is better because it contains words referring to very similar concepts: a bar of uranium is nuclear combustible. Another example is the following, for the verb "to attribute“:

\begin{tabular}{|l|l|l|}
\hline attribuer & $C O D$ & $\begin{array}{l}\text { parole, prix, décoration, pape, responsabilité, télévision, } \\
\text { attentat, lettre, contrat, ministre, jury, fond, autorité, note, } \\
\text { bonus, bande, bombardement }\end{array}$ \\
\hline attribuer & $C O D$ & prix, décoration \\
\hline to attribute & object & $\begin{array}{l}\text { talk, prize, decoration, pope, responsibility, television, } \\
\text { attempt, letter, contract, ministry, jury, funds, authority, note, } \\
\text { bonus, band, bombing }\end{array}$ \\
\hline to attribute & object & prize, decoration \\
\hline
\end{tabular}

Obtaining meaningful classes with a corpus such as "AFP" shows the efficiency of our method. Moreover, we obtain cohesive classes for verbs very general and polysemous.

Words are often polysemous or ambiguous. However, when used in context, they denote one meaning, and moreover this meaning is generally the same in different occurrences of a same context. When building classes according to their context, we avoid mixing all the meanings of a word in a same class. Such a result can be exhibited in the classes (law, constitution) and (law, article, disposition) in the juridical context, for the words "articles", "constitution" and "disposition".

\section{$6 \quad$ Related Works}

There is a lot of works dedicated to the formation of classes of words. These classes have very various statuses. They can contain words belonging to the same semantic field or near synonymous. We give here some details about three approaches we cited in the introduction that are good examples of different approaches: manual, statistical and syntactical.

WordNet [7] is a lexical database made by lexicographers. It aims at representing the sense of the bigger part of the lexicon. It is composed of Synsets. A Synset is a set of words that are synonymous. These Synsets are linked by IS A relations. Its coverage 
is large but this quality is, in a sense, its shortcoming. Indeed, the generality of its contents makes it difficult to use in real sized applications that are often centered on a domain. It rarely can be used without a lot of manual adaptation, even if some authors tried to relate automatically acquired lexical knowledge with it, for example [2].

IMToolset, by Uri Zernik [15], extracts, for a word, several clusters of words from text. Each of these clusters reflects a different meaning of the studied word. This extraction is done by scanning the local contexts of the word, the 10 words surrounding it in the texts. These signatures are statistically analyzed and clustered. The result is groups of words that are similar to our domains but more focused on the sense of a word alone.

We have already stressed out some characteristics of ASIUM by D. Faure and C. Nedellec, but we give here some more details. ASIUM learns subcategorization frames of verbs and ontologies from text using syntactic analysis and a conceptual clustering algorithm. It analyses texts with Sylex and creates basic clusters of words appearing with a same verb and a same syntactic role or preposition, as do SVETLAN'. These basic classes are then clustered to create an ontology by the mean of a cooperative learning algorithm. The main difference with SVETLAN' is this cooperative part: ASIUM critically depends on the expert that has to valid, and possibly split, the clusters made by the algorithm. As a consequence, it has to work on specific technical texts. As a consequence, ASIUM, applied on texts such as our AFP wires would certainly not have been able to extract good classes. Indeed, there is a lot of very polysemous words in these texts that take a precise meaning only in a sufficiently focused context, such as our domains. Furthermore, as each word does not occur a lot, the distance measure currently used in ASIUM would not allow grouping any cluster.

\section{$7 \quad$ Future Directions of Work}

We envisage two major directions of work that are closely related: the extension of our results and a real validation of them.

At this time, the classes do not contain a lot of words. A way to enlarge them, and the more obvious one, is to process more texts in order to have more data. That will necessitate some modifications of the algorithm of domain building. At this time, we store all the thematic units and all the words in domains, even if they are never aggregated and so have always an occurrence number of one. Thus, the memory usage is too important. We will set up a kind of "garbage collection" process to remove or maybe recycle old and not very aggregated domains. This will allow us to process much more texts and so enlarge classes in two directions: their sizes and the number of occurrences of words inside them.

Another way could be to regroup classes that are related to the same verb, by the same syntactic relation in two domains belonging to the same hierarchy, i.e. a same more general context, assuming the words always have the same meaning. However this method has to be tested on more results in order to prove its reliability. With our results, we would build for example (law, constitution, article, disposition) in the domain of "Law" and (rebel, force, northerner, leader) in the domain of "conflict". This way of enlarging the classes should be studied carefully in a theoretical point of view. Indeed, the originality of our work is the focused context entailed by the use of 
the domains. That allows us to deal with texts from general language. When we consider domains in the same hierarchy, we enlarge the context and so, we take the risk to have the same problems as other works, discussed in the introduction and Sect. 6, when they are applied to general language: the merging in a same class of different meanings of some words.

Another possibility to enlarge our classes is to generalize them as in ASIUM. ASIUM merges classes independently of the related verbs according to a similarity measure, even if, in our case, this generalization process would operate in a same general domain. Afterwards, as in ASIUM, we would ask an expert to validate its results. The problem would be, again, to find an expert of "general language".

Concerning the validation task, it could be realized by relating our classes to existing classifications, as do [2] with WordNet. Another possibility would be to evaluate the improvement SVETLAN' permits when performing a task. Our group participates to the "Question Answering" track in the TREC evaluations and uses a search engine to find documents able to contain the answers. The questions of the QA track are about various topics from a newspaper corpus. So, we believe that the classes obtained by SVETLAN' could be very useful to extend precisely the requests to the search engine.

\section{Conclusion}

The system SVETLAN' we propose, in conjunction with SEGAPSITH and the syntactic parser Sylex, extracts classes of words from raw text. Instead of just having sets of weighted words for describing semantic domains, domains are described by a set of verbs related to classes of words by a syntactic link. These classes are created by the gathering of nouns appearing with the same syntactic role after the same verb inside a context. This context is made by the aggregation of text about similar subjects. Besides, we can also view this base as semantic classes, each one being related to its context of interpretation. The first experiments carried out give satisfying results. But they also confirm that a great volume of data is necessary in order to extract a large quantity of lexical knowledge by the analysis of syntactic distributions. Moreover the very low recall of the syntactic parser and its systematic errors on some constructions, for example the passive form, which is very common in the journalistic style of our corpus, reduce the number and size of the classes. To solve this problem, we envisage trying another analyzer or adding a post-processing step to Sylex that detects the passive form by using data already in its output. These adaptations and the study of larger corpora will allow us to obtain a good coverage of numerous semantic domains. So, we will be able to give valuable semantic data useful in a lot of applications as information retrieval systems or word sense disambiguation systems.

\section{References}

1. Basili, R., Pazienza, M.T., Velardi, P.: What can be learned from raw texts? Machine Translation, Vol. 8 (1993) 147-173

2. Basili, R., Della Rocca, M., Pazienza, M.T., Velardi, P.: Contexts and Categories: Tuning a 
General Purpose Verb Classification to Sublanguages. Proceedings of the International Conference on Recent Advances in Natural Language Processing, Tzigov Chark, Bulgaria, (14-16 Sept.1995)

3. Constant, P.: Analyse Syntaxique Par Couches. PhD thesis, École Nationale Supérieure des Télécommunications, (Apr. 1991)

4. Constant, P.: L'analyseur linguistique SYLEX. 5ème école d'été du CNET (1995).

5. Fabre, C., Habert, B., Labbé, D.: La polysémie dans la langue générale et les discours spécialisés. Sémiotiques, number 13 (1997) 15-31

6. Faure, D., Nedellec, C.: ASIUM, Learning subcategorization frames and restrictions of selection. In: Kodratoff, Y. (ed.): Proceedings of the $10^{\text {th }}$ European Conference on Machine Learning - Workshop on text mining (1998)

7. Fellbaum, C. (Ed.): WordNet: an electronic lexical database. The MIT Press (1998)

8. Ferret, O.: How to thematically segment texts by using lexical cohesion? Proceedings of ACL-COLING'98 (student session). Montreal, Canada, (1998) 1481-1483

9. Ferret, O., Grau, B.: A Thematic Segmentation Procedure for Extracting Semantic Domains from Texts. Proceedings of the European Conference on Artificial Intelligence, ECAI'98. Brighton, UK (1998)

10.Greffenstette, G.: Explorations in automatic thesaurus discovery. Kluwer Academic Pub., Boston (1994)

11.Habert, B., Fabre, F.: Elementary dependency trees for identifying corpus-specific semantic classes. Computers and the Humanities, volume 33, number 3 (1999) 207-219

12.Lin, C.-Y.: Robust Automated Topic Identification. Doctoral Dissertation, University of Southern California (1997)

13.Pereira, F., Tishby, N., Lee, L.: Distributional clustering of English words. Proceedings of ACL'93 (1993)

14.Rousselot, F., Frath, P., Oueslati, R.: Extracting concepts and relations from corpora. Proceeding of the Corpus-Oriented Semantic Analysis workshop of the European Conference on Artificial Intelligence, ECAI'96. Budapest, Hungary (1996) 74-78

15.Zernik, U.: TRAIN1 vs. TRAIN2: Tagging Word Senses in Corpus. Proceedings of Recherche d'Informations Assistée par Ordinateur, RIAO'91 (1991) 